\title{
Research Article \\ Optimum Maintenance Policy of a Periodically Inspected System under Imperfect Repair
}

\author{
F. G. Badía and M. D. Berrade \\ Departamento de Métodos Estadísticos, Centro Politécnico Superior de Ingenieros, \\ Universidad de Zaragoza, 50018 Zaragoza, Spain \\ Correspondence should be addressed to F. G. Badía, gbadia@unizar.es
}

Received 19 November 2008; Accepted 21 April 2009

Recommended by Shey-Huei Sheu

This paper deals with the maintenance policy of a system subject to periodical inspections aimed at detecting the occurrence of failures. After the first $N-1$ failure, the system undergoes an imperfect repair that brings the system back to the operating condition while the $N$ th failure is followed by a perfect repair restoring the unit to an as-good-as-new condition. The limiting average availability as well as the minimum cost policy are discussed.

Copyright (C) 2009 F. G. Badía and M. D. Berrade. This is an open access article distributed under the Creative Commons Attribution License, which permits unrestricted use, distribution, and reproduction in any medium, provided the original work is properly cited.

\section{Introduction}

All the systems can undergo random failures. In some cases these may cause disastrous consequences in human life, being the nuclear plants the most outstanding example. The occurrence of failures in manufacturing systems devoted to the production of goods has not so devastating effects but causes, in general, economic losses due to the downtime and the lack of system availability.

Maintenance policies are mainly concerned with age and usage, both being responsible of system failure. The preventive maintenance is carried out previous to failure and aims at reducing breakdown risk. The corrective maintenance is performed after system failure and can be classified into perfect repair or imperfect repair. The former brings the system back to an as-good-as-new condition by any procedure or even the whole replacement of the system by a new identical one. The latter restores the operating condition somewhere between as-bad-as-old and as-good-as-new. Whenever the whole system is not replaced but only some of its components, the imperfect repair constitutes a more realistic approach. In the works due to Brown and Proschan [1] as well as Nakagawa and Yasui [2] an imperfect maintenance is achieved with probability $p$ or a perfect one with probability $1-p$. It is 
important to remark that different probabilistic structures emerge depending on the quality of maintenance actions.

Moreover, many engineering systems are subject to the so-called unrevealed failures, that is, those that are detected only by special tests or inspection. Failures of this sort are typical in systems that are not in continuous operation such as spares or units in stand-by mode. If the failure happens while the mechanism is in an idle period it will remain undiscovered until the following attempt of use unless the system is monitored. The unaffordable cost of a continuous monitoring motivates the periodic inspection of the system which is well worth doing. Several works focus on bivariate policies based on both periodic inspections at times $k T, k=1,2 \ldots$ to detect the otherwise unrevealed failures, along with the accumulated number of failures, $N$. The works of Badía and Berrade [3-5], Zequeira and Bérenger [6], Zhang et al. [7], Wang and Zhang [8] deal with bivariate policies that aim at getting the optimum policy minimizing the long-run expected cost per unit of time.

Some examples of periodically inspected systems are detectors of fire, gas, as well as pressure and safety valves installed to prevent special risks. In fact, Vaurio [9] indicates that unrevealed failures are quite common in safety systems of nuclear plants. Once the failure has been discovered, the system can undergo both an imperfect repair or a perfect one. The former is usually less expensive although after an imperfect repair the system lifetime commonly appears to be shorter than when a perfect restoration is carried out. Therefore, several imperfect repairs are allowed previously to the perfect repair or the eventual replacement of the unit. Such imperfect repairs try to prolong system lifetime as much as possible. Biswas et al. [10] analyze the availability function of a periodically inspected system that experiences a fixed number of imperfect repairs before being perfectly repaired. Zhang et al. [7], and Wang and Zhang [8] focus on optimum bivariate policies where both parameters, the periodic times for preventive maintenance and the accumulated number of failures foregoing the replacement of the system are considered. In this work we aim at designing a bivariate policy that takes into account the inspection times along with the allowed number of failures before the perfect repair, studying both the system availability and maintenance optimization. Our goal is the cost minimization over an infinite time span as well as obtaining conditions under which there exists an optimum policy. The maintenance model along with the availability function are presented in the second section. The cost function along with the main results concerning the existence of an optimum policy are in the third section where the relevant conclusions are also provided. The last section contains some examples which illustrate the theoretical results.

\section{Maintenance Model and Availability}

Consider a system whose failures belong to the unrevealed type which is in operation at $t=0$ and tested at times $n T, n=1,2, \ldots$ to check whether a failure has taken place. If the system fails during the time span between two consecutive inspections this failure will remain undetected until the following inspection after which the system undergoes an imperfect repair. When the $N$ th failure is detected a perfect repair is carried out. Hence, $N-1$ imperfect repairs are carried out before the perfect repair. In this model times of inspections are considered negligible but times of repairs will be taken into account. In what follows the notation below will be used. 
(i) $X_{j}, j=1,2, \ldots, N$ : time to next failure after the $j-1$ imperfect repair.

(ii) $\bar{F}_{j}, j=1,2, \ldots, N$ : reliability function corresponding to $X_{j}$.

(iii) $\mu_{j}=E\left[X_{j}\right]=\int_{0}^{\infty} \bar{F}_{j}(x) d x, j=1,2, \ldots, N$.

(iv) $M_{N}=\sum_{j=1}^{N} \mu_{j}$.

(v) $K_{j}, j=1,2, \ldots, N$ : number of inspections after the $j-1$ failure is detected until the $j$ failure occurs. Therefore $K_{j}=\left\lceil X_{j} / T\right\rceil$, where \lceil\rceil denotes the integer part function.

(vi) $E\left[K_{j}\right]=\sum_{n=1}^{\infty} \bar{F}_{j}(n T)=S_{j}(T), j=1,2, \ldots, N$.

(vii) $H_{N}(T)=\sum_{j=1}^{N} S_{j}(T)$.

(viii) $R_{j}, j=1,2, \ldots, N$ : time of the $j$ repair.

(ix) $r_{j}=E\left[R_{j}\right], j=1,2, \ldots, N$.

(x) $d_{N}=\sum_{j=1}^{N} r_{j}$.

We assume that the mean times of repair, $r_{j}$ constitutes an increasing sequence in $j$. In addition, the perfect repair restores the system to an as-good-as-new condition. Nevertheless system deterioration cannot be removed by imperfect repairs and it is assumed that the system lifetimes after them show a decreasing pattern. Therefore the following stochastic order relations hold:

$$
X_{1} \geq X_{2} \geq \stackrel{s t}{s t} \stackrel{s t}{\geq}_{N}
$$

where st means the usual stochastic order, that is,

$$
\bar{F}_{1}(u) \geq \bar{F}_{2}(u) \geq \cdots \geq \bar{F}_{N-1}(u) \geq \bar{F}_{N}(u)
$$

A cycle denoted by $\tau$ represents the time span between two consecutive renewals of the system (see Ross [11]). In this case a cycle is completed whenever a perfect repair is carried out. Therefore, its length is given as follows:

$$
\tau=\sum_{j=1}^{N}\left[\left(K_{j}+1\right) T+R_{j}\right],
$$

and its expected length

$$
E[\tau]=T\left(H_{N}(T)+N\right)+d_{N}
$$

The availability function at $t$ denoted by $A(t)$, provides the probability that the system is ready to function whether there is an attempt of use at time $t$. In many practical situations the limiting average availability is used instead:

$$
A(T, N)=\lim _{t \rightarrow \infty} \frac{1}{t} \int_{0}^{t} A(u) d u=\frac{E[U]}{E[U]+E[D]},
$$


where $E[U]$ and $E[D]$ represent, respectively, the expected uptime and downtime during a cycle. Moreover

$$
E[U]+E[D]=E[\tau]
$$

In this case the foregoing expression turns out to be

$$
A(T, N)=\frac{M_{N}}{T\left(H_{N}(T)+N\right)+d_{N}} .
$$

We should refer the reader to the following properties of the $S_{j}(T)$ shown in Badía et al. [12]:

$$
\begin{aligned}
& S_{j}(T) \text { is a decreasing function, } \\
& \lim _{T \rightarrow 0} S_{j}(T)=\infty, \\
& \lim _{T \rightarrow \infty} S_{j}(T)=\lim _{T \rightarrow \infty} T S_{j}(T)=0, \\
& \lim _{T \rightarrow 0} T S_{j}(T)=\mu_{j} .
\end{aligned}
$$

Therefore, the next conditions also hold

$$
\lim _{T \rightarrow 0} A(T, N)=\frac{M_{N}}{M_{N}+d_{N}}, \quad \lim _{T \rightarrow \infty} A(T, N)=0 .
$$

The limit on the right implies that systems of this sort under no inspection tend to be unavailable.

Furthermore, $A(T, N)$ is a decreasing function with $T$ if and only if $T\left(H_{N}(T)+N\right)+d_{N}$ is an increasing one. The derivative of the latter is expressed as

$$
l(T, N)=\frac{d\left(T\left(H_{N}(T)+N\right)+d_{N}\right)}{d T}=T \frac{d H_{N}(T)}{d T}+H_{N}(T)+N
$$

\section{The exponential case}

Now, let us assume that the sequence of times between failures, $X_{i}, i=1,2, \ldots, N$ are exponentially distributed with mean value $1 / \lambda_{i}, i=1,2, \ldots, N$ and $\lambda_{1} \leq \lambda_{2} \leq \cdots \leq \lambda_{N}$. Then

$$
\begin{aligned}
S_{j}(T) & =\frac{1}{e^{\lambda_{j} T}-1}, \\
H_{N}(T) & =\sum_{j=1}^{N} \frac{1}{e^{\lambda_{j} T}-1},
\end{aligned}
$$


hence

$$
l(T, N)=\sum_{j=1}^{N} e^{\lambda_{j} T} \frac{\left(e^{\lambda_{j} T}-1-\lambda_{j} T\right)}{\left(e^{\lambda_{j} T}-1\right)^{2}} \geq 0
$$

Therefore, the system shows a decreasing availability. Thus, the less frequent the inspection, the more likely the system to be unavailable when required for use.

\section{Cost Function and Optimum Maintenance Policy}

In this section we focus on maintenance optimization. The model includes the following costs.

(i) $c_{1}$ : unitary cost of inspection.

(ii) $c_{r j}$ : unitary cost caused by the imperfect repair of the $j$ th failure, $j=1,2, \ldots, N-1$, $c_{r j}$ is assumed to be an increasing sequence in $j$.

(iii) $c_{r p}$ : cost of the perfect repair.

(iv) $c_{N}$ : total cost incurred in a cycle due to repairs, $c_{N}=\sum_{j=1}^{N-1} c_{r j}+c_{r p}$.

(v) $c_{d}$ : cost rate due to downtime.

The cost per unit of time over an infinite time span, denoted by $Q(T, N)$, is assumed to be the cost function of this model. $Q(T, N)$ depends on the time interval between periodic inspections, $T$, as well as on the number of failures previous to the perfect repair, $N$.

The key theorem of the renewal-reward theory (see Ross [11]) ensures that, as time tends to infinity, $Q(T, N)$ converges to the ratio of the expected cost of a cycle and its expected length:

$$
Q(T, N)=\frac{E[C(\tau)]}{E[\tau]}
$$

The cost of a cycle is given as follows:

$$
C(\tau)=c_{1} \sum_{j=1}^{N}\left(K_{j}+1\right)+c_{N}+c_{d}\left\{\sum_{j=1}^{N}\left(K_{j}+1\right) T+R_{j}-X_{j}\right\}
$$

The three terms in the previous expression correspond to the cost incurred due to inspections, repairs and the downtime cost, respectively.

The expected cost of a cycle is

$$
E[C(\tau)]=c_{d}\left[T\left(H_{N}(T)+N\right)+d_{N}\right]+V_{N}+c_{1} H_{N}(T),
$$

where

$$
V_{N}=N c_{1}+c_{N}-c_{d} M_{N}
$$




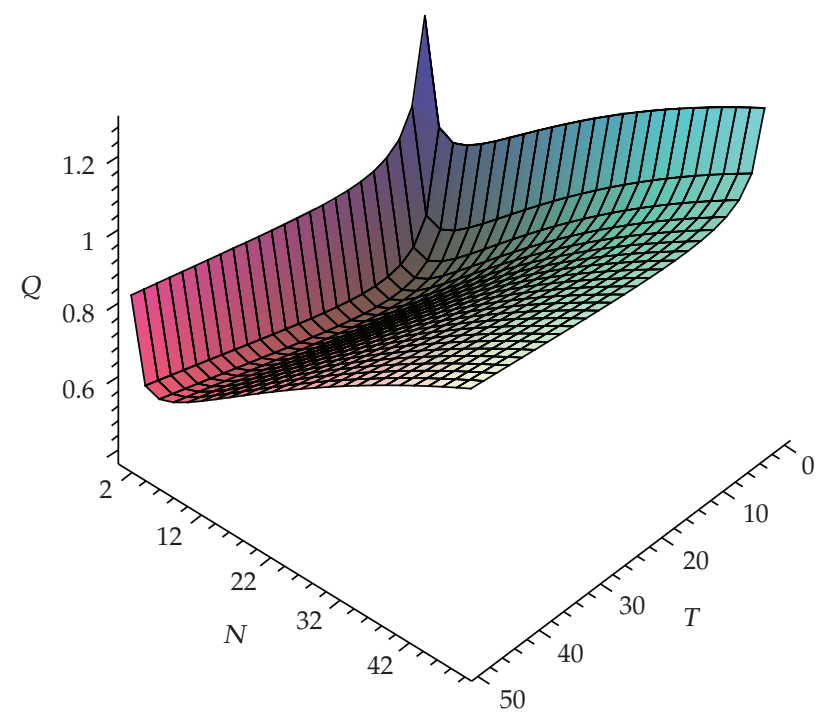

Figure 1: Cost function. $Q(T, N)$.

The cost function turns out to be

$$
Q(T, N)=c_{d}+\frac{V_{N}+c_{1} H_{N}(T)}{T\left(H_{N}(T)+N\right)+d_{N}} .
$$

Figure 1 shows $Q(T, N)$ under the following costs: $c_{1}=1, c_{r j}=2 j,(j=1, \ldots), c_{r p}=50, c_{d}=$ $2, r_{j}=0.25 j(j=1, \ldots)$. The time between consecutive failures is assumed to follow an exponential distribution with parameter $\lambda=0.01$.

The following result aim at providing conditions for the existence of an optimum inspection interval when the number of imperfect inspections previous to the perfect one is fixed. The key of the proof rests on the fact that, whenever the condition $V_{N}<0$ holds, the cost function is $U$-shaped, that is, first decreases, reversing this condition as time goes by.

Theorem 3.1. Given $N$, there exists $T^{\star}, 0<T^{\star}<\infty$, minimizing $Q(T, N)$, if and only if $V_{N}<0$.

Proof. The same properties concerning $S_{j}(T)$ that appear in Section 2 are used to prove that $Q(T, N)$ verifies the limiting conditions below:

$$
\lim _{T \rightarrow 0} Q(T, N)=\infty, \quad \lim _{T \rightarrow \infty} Q(T, N)=c_{d}
$$

$V_{N}+c_{1} H_{N}(T)$ is a continuous and nonincreasing function. The same conditions of Section 2 along $V_{N}<0$ imply that there exists $T_{0} \in(0, \infty)$ with $T_{0}$ being the unique solution of the following equation:

$$
V_{N}+c_{1} H_{N}(T)=0
$$


In addition

$$
Q(T, N)<c_{d}, \quad \text { for all } T>T_{0} .
$$

The existence of a minimum, $T^{\star}$, is deduced from the continuity of $Q(T, N)$ along with $\lim _{T \rightarrow \infty} Q(T, N)=c_{d}$.

Moreover $T^{\star}$ is one of the roots of the next equation:

$$
\frac{d H_{N}(T)}{d T}\left(c_{1} T N+c_{1} d_{N}-V_{N} T\right)-\left(V_{N}+c_{1} H_{N}(T)\right)\left(H_{N}(T)+N\right)=0
$$

In case that $V_{N} \geq 0$, then

$$
Q(T, N) \geq c_{d}=\lim _{T \rightarrow \infty} Q(T, N)
$$

therefore, $T^{\star}=\infty$.

The critical condition $V_{N}<0$ is equivalent to $M_{N}>\left(N c_{1}+c_{N}\right) / c_{d}$, that is, it is worth carrying out a maintenance policy as long as the mean time previous to the perfect restoration is long enough. Otherwise, the maintenance costs do not compensate the benefits derived from them.

\subsection{Optimum $\mathbf{N}$}

The maintenance policy consists of imperfect repairs following the $N-1$ first failures along with a perfect repair after the $N$ th failure. The purpose of this section is to seek the accumulated number of failures, $N$, minimizing $Q(T, N)$.

We denote by $N^{\star}$ the optimal $N$. The following conditions assure the existence of a local minimum:

$$
Q\left(T, N^{\star}+1\right) \geq Q\left(T, N^{\star}\right), \quad Q\left(T, N^{\star}-1\right)>Q\left(T, N^{\star}\right) .
$$

$Q(T, N)$ is also expressed as follows:

$$
Q(T, N)=c_{d}+\frac{a(T, N)}{b(T, N)}
$$

where

$$
\begin{gathered}
a(T, N)=V_{N}+c_{1} H_{N}(T), \\
b(T, N)=T\left(H_{N}(T)+N\right)+d_{N} .
\end{gathered}
$$


Moreover

$$
\begin{gathered}
a(T, N+1)=a(T, N)+B_{N}+c_{1} S_{N+1}(T), \\
b(T, N+1)=b(T, N)+T\left(S_{N+1}(T)+1\right)+r_{N+1},
\end{gathered}
$$

with

$$
B_{N}=c_{1}+c_{r N}-c_{d} \mu_{N+1}
$$

$Q(T, N+1) \leq Q(T, N)$ is equivalent to the following inequality:

$$
c_{d}+\frac{B_{N}+c_{1} S_{N+1}(T)}{T\left(S_{N+1}(T)+1\right)+r_{N+1}} \leq c_{d}+\frac{V_{N}+c_{1} H_{N}(T)}{T\left(H_{N}(T)+N\right)+d_{N}}=Q(T, N)
$$

From the stochastic order defined in (2.2) by the magnitude of tail probabilities, it follows that

$$
S_{1}(T) \geq S_{2}(T) \geq \cdots \geq S_{N-1}(T) \geq S_{N}(T)
$$

Hence $H_{N}(T) \geq N S_{N+1}(T)$. In addition $Q(T, N)$ is an increasing function with $H_{N}(T)$ if and only if $V_{N}<0$, therefore

$$
Q(T, N) \geq c_{d}+\frac{V_{N} / N+c_{1} S_{N+1}(T)}{T\left(S_{N+1}(T)+1\right)+d_{N} / N}
$$

Consider now identical repair times, that is,

$$
r_{1}=r_{2}=\cdots=r_{N}=r_{N+1}=\cdots,
$$

then $d_{N} / N=r_{N+1}$. Hence, the inequality in (3.16) is satisfied provided that $B_{N} \leq V_{N} / N$ holds.

From the foregoing calculations we derive that in case of $B_{N} \leq V_{N} / N$ then $Q(T, N+$ $1) \leq Q(T, N)$. Moreover if $B_{N} \leq V_{N} / N$ for all $N$, then $N^{\star}=\infty$.

The condition $B_{N}>V_{N} / N$ is also expressed as

$$
\mu_{N+1}<\frac{N c_{r N}+c_{d} M_{N}-c_{N}}{N c_{d}}
$$

Hence $N^{\star} \geq N_{0}$ where $N_{0}$ is defined below:

$$
N_{0}=\min \left\{N: \mu_{N+1}<\frac{N c_{r N}+c_{d} M_{N}-c_{N}}{N c_{d}}\right\} .
$$


It's important to remark that $N B_{N}-V_{N}$ is an increasing function, so it can be stated that

$$
N_{0}=\min \left\{N: N B_{N}-V_{N}>0\right\}
$$

Condition $B_{N}>V_{N} / N$ and the equivalent expression in (3.20) are concerned with the existence of a finite $N^{\star}$. They indicate that the system should undergo a perfect restoration provided that the remaining time to the next failure does not exceed the upper bound in (3.20). That limit turns out to be a relation involving maintenance costs and past times between failures, stating the instant when the perfect repair, that is, the change by a new system, constitutes an optimal choice from the cost view point.

\subsection{Exponential Failure Time and Minimal Repair}

We assume now that the first time to failure follows an exponential distribution and the imperfect repair consists of minimal repair that restores the system to the operating state just previous to failure. Hence all the successive times between failures, $X_{j}$, are also exponentially distributed with $\mu_{j}=1 / \lambda$ being its mean value.

In this case

$$
S_{j}(T)=\frac{1}{e^{\lambda T}-1}, \quad H_{N}(T)=\frac{N}{e^{\lambda T}-1} .
$$

The cost function is given next:

$$
Q(T, N)=c_{d}+\frac{V_{N} / N+c_{1} /\left(e^{\lambda T}-1\right)}{T\left(1 /\left(e^{\lambda T}-1\right)+1\right)+d_{N} / N^{\prime}}
$$

with $V_{N}=N c_{1}+c_{N}-c_{d} N / \lambda$.

In what follows we aim at obtaining the optimum policy denoted by $\left(T^{\star}, N^{\star}\right)$, satisfying that

$$
Q\left(T^{\star}, N^{\star}\right)=\min _{T, N} Q(T, N)=\min _{N} Q\left(T_{N}^{\star}, N\right)
$$

where $T_{N}^{\star}$ represents the optimum $T$ for a given $N$.

We can restrict the analysis to the $V_{N}<0$ case, as whenever $V_{N} \geq 0$, then $T_{N}^{\star}=\infty$ and $Q\left(T_{N}^{\star}, N\right)=c_{d} \geq \min _{N} Q\left(T_{N}^{\star}, N\right)$.

The goal of the following analysis is to prove that

$$
\min _{N} Q\left(T_{N}^{\star}, N\right)=\min _{N \leq N_{0}} Q\left(T_{N}^{\star}, N\right),
$$

with $N_{0}=\min \left\{N: N B_{N}-V_{N}>0\right\}=\min \left\{N: c_{r N}>\left(\sum_{j=1}^{N-1} c_{r j}+c_{p r}\right) / N\right\}$. 
Given $N$ and provided that $V_{N}<0$, the optimum in $T, T_{N}^{\star}$ is the only root of the equation $M(T, N)=0$ where

$$
M(T, N)=\left(-\frac{V_{N}}{N}\right) e^{\lambda T}-\left(-\frac{V_{N}}{N}+c_{1}\right)-\left(-\frac{V_{N}}{N}+c_{1}\right) \lambda T-c_{1} \lambda \frac{d_{N}}{N} .
$$

A close inspection of $M(T, N)$ lets us conclude that in case that $N \geq N_{0}$, then $T_{N}^{\star} \leq T_{N+1}^{\star}$.

In addition $Q(T, N) \leq Q(T, N+1)$ if and only if $R(T, N) \geq 0$, where $R(T, N)$ is defined next:

$$
R(T, N)=B_{N} d_{N}-V_{N} r_{N+1}+\left(N B_{N}-V_{N}\right) \frac{T e^{\lambda T}}{e^{\lambda T}-1}+c_{1}\left(d_{N}-N r_{N+1}\right) \frac{1}{e^{\lambda T}-1} .
$$

$R(T, N)$ is an increasing function in $T$ for $N \geq N_{0}$. Moreover

$$
R\left(T_{N+1}^{\star}, N+1\right)-R\left(T_{N+1}^{\star}, N\right) \geq 0
$$

provided that $V_{N+1}<0$. Therefore, if $N \geq N_{0}$ then

$$
\begin{aligned}
R\left(T_{N+1}^{\star}, N+1\right) & \geq R\left(T_{N+1}^{\star}, N\right) \\
& \geq R\left(T_{N}^{\star}, N\right) \\
& \geq\left(\frac{V_{N}}{N}+c_{1} \frac{1}{e^{\lambda T_{N}^{\star}-1}}\right)\left(d_{N}-N r_{N+1}\right)+\left(N B_{N}-V_{N}\right) \frac{T_{N}^{\star} e^{\lambda T_{N}^{\star}}}{e^{\lambda T_{N}^{\star}-1}} \\
& \geq 0 .
\end{aligned}
$$

Next, we show that $Q\left(T_{N_{0}}^{\star}, N_{0}\right) \leq Q\left(T_{N^{*}}^{\star}, N\right)$ for $N \geq N_{0}$. The following facts hold, namely, $R(T, N)$ is increasing in $T$ and $T_{N}^{\star}>T_{N_{0}}^{\star}$. Then $R\left(T_{N^{\prime}}^{\star}, N_{0}\right) \geq R\left(T_{N_{0}}^{\star}, N_{0}\right) \geq 0$. Therefore, $Q\left(T_{N}^{\star}, N_{0}\right) \leq Q\left(T_{N}^{\star}, N_{0}+1\right)$. The same arguments lead to prove that $Q\left(T_{N}^{\star}, N_{0}+1\right) \leq$ $Q\left(T_{N^{*}}^{\star}, N_{0}+2\right)$ as well as $Q\left(T_{N^{*}}^{\star}, N_{0}\right) \leq Q\left(T_{N^{*}}^{\star}, N\right)$ for $N \geq N_{0}$. Therefore $Q\left(T_{N_{0}}^{\star}, N_{0}\right) \leq$ $Q\left(T_{N}^{\star}, N_{0}\right) \leq Q\left(T_{N}^{\star}, N\right)$ for $N \geq N_{0}$. Hence, it follows that

$$
\min _{N} Q\left(T_{N}^{\star}, N\right)=\min _{N \leq N_{0}} Q\left(T_{N}^{\star}, N\right)
$$

Moreover, in case that we have identical repair times, the foregoing result along with the one in Section 3.1 leads to

$$
Q\left(T^{\star}, N^{\star}\right)=\min _{T, N} Q(T, N)=\min _{N} Q\left(T_{N}^{\star}, N\right)=Q\left(T_{N_{0}}^{\star}, N_{0}\right) .
$$

\subsection{Concluding Remarks}

This model constitutes an approach to the common maintenance practice consisting of several repairs during the useful life of a system that end with the change by a new one when 
the expected time to be usable is not long enough. By means of the stochastic order in the successive times to failure and increasing repair times we reflect that, as time goes by, the system condition becomes less than perfect with progressively higher maintenance costs.

A system subject to inspection to detect its failures is considered, and thus we deal with a bivariate policy where one of the terms refers to the inspection time and the other to the number of failures previous to the system substitution. Regarding the optimum policy we obtain conditions ensuring when inspections and repairs of the system in use are still advantageous.

The study is completed with some insights related to times between failures following exponential distributions.

\section{Examples}

Example 4.1. Let's consider that $X_{j} j, j=1,2, \ldots$ is a Weibull random variable whose distribution function is given as follows:

$$
\bar{F}_{j}(x)=e^{-\lambda_{j} x^{2}}, \quad x \geq 0,
$$

with $\lambda_{j}=j \lambda$ and $\lambda>0$. The mean time to the $j$ th failure after the $j-1$ imperfect repair is

$$
\mu_{j}=\frac{1}{2} \sqrt{\frac{\pi}{\lambda_{j}}}
$$

We assume the following imperfect repair cost of the $j$ th failure

$$
c_{r j}=\min (j c, 10 c),
$$

with $c$ being a positive constant. In addition the time of the imperfect $j$ th repair is set $r_{j}=0.1$.

It's known from results in Section 3.1, that the optimum $N$ is greater or equal to $N_{0}=$ $\min \left\{N: N B_{N}-V_{N}>0\right\}$. We aim at obtaining a local optimum for both $T$ and $N$ by means of the following algorithm proposed in Nakagawa [13] as well as in Zequeira and Bérenguer [6].

Step 1. Set $N^{\star}=N_{0}$.

Step 2. Calculate $T_{N^{\star}}^{\star} \mathrm{y} T_{N^{\star}+1}^{\star}$.

Step 3. If $Q\left(T_{N^{\star}+1}^{\star}, N^{\star}+1\right) \leq Q\left(T_{N^{\star}}^{\star}, N^{\star}\right)$ then set $N^{\star}=N^{\star}+1$ and go to Step 2 .

Table 1 contains the values of $N_{0}$ along with the local optimum $N^{\star}$ and $T_{N^{\star}}^{\star}$ under different values of the costs and the parameters $c$ and $\lambda$.

Example 4.2. Consider a system whose time to failure is assumed to be an exponential distribution with mean equal to 1 and subject to inspections with unitary cost $c_{1}=1$ as well as minimal repairs with costs $c_{r j}=\alpha j, j=1,2 \ldots N-1$. The cost due to downtime is $c_{d}=10$. 
Table 1: Local optimum $\mathbf{N}^{\star}$ and $\mathbf{T}_{\mathbf{N}^{\star}}^{\star}$.

\begin{tabular}{ccccccccc}
\hline$c_{1}$ & $c$ & $c_{p r}$ & $c_{d}$ & $\lambda$ & $N_{0}$ & $N^{\star}$ & $T_{N^{\star}}^{\star}$ & $Q\left(T_{N^{\star}}^{\star}, N^{\star}\right)$ \\
\hline 1 & 2 & 10 & 2 & 0.001 & 2 & 3 & 27.9146 & 1.1790 \\
1 & 4 & 50 & 2 & 0.001 & 3 & 3 & 28.3133 & 2.4766 \\
1 & 5 & 150 & 3.5 & 0.001 & 5 & 5 & 24.1634 & 2.8601 \\
\hline
\end{tabular}

Table 2: $\mathbf{N}^{\star}, \mathbf{T}^{\star}, \mathbf{Q}\left(\mathbf{T}^{\star}, \mathbf{N}^{\star}\right)$. Costs: $\mathbf{c}_{\mathbf{r j}}=\mathbf{j}, \mathbf{j}=1,2 \ldots \mathbf{N}-1$.

\begin{tabular}{lcccc}
\hline$c_{p r}$ & $N_{0}$ & $N^{\star}$ & $T^{\star}$ & $Q\left(T^{\star}, N^{\star}\right)$ \\
\hline 8 & 4 & 4 & 0.8145 & 7.1165 \\
12 & 5 & 5 & 0.9131 & 7.6919 \\
15 & 6 & 5 & 0.9786 & 8.0532 \\
20 & 6 & 6 & 1.12053 & 8.5470 \\
25 & 7 & 7 & 1.2953 & 8.9599 \\
30 & 8 & 8 & 1.5301 & 9.3132 \\
\hline
\end{tabular}

Table 3: $\mathbf{N}^{\star}, \mathbf{T}^{\star}, \mathbf{Q}\left(\mathbf{T}^{\star}, \mathbf{N}^{\star}\right)$. Costs: $\mathbf{c}_{\mathbf{p r}}=15$.

\begin{tabular}{lcccc}
\hline$\alpha$ & $N_{0}$ & $N^{\star}$ & $T^{\star}$ & $Q\left(T^{\star}, N^{\star}\right)$ \\
\hline 0.5 & 8 & 7 & 0.8854 & 7.2551 \\
0.7 & 7 & 6 & 0.9193 & 7.6140 \\
1.2 & 5 & 4 & 1.0290 & 8.7000 \\
1.5 & 5 & 4 & 1.1025 & 8.9000 \\
2.5 & 4 & 3 & 1.5065 & 9.9511 \\
3 & 3 & 3 & 1.8123 & 9.6981 \\
\hline
\end{tabular}

The times for repair are given as $r_{j}=0.25 j, j=1,2 \ldots$ Table 2 shows the optimum policy under different costs of the perfect repair $c_{p r}$ whereas Table 3 deals with distinct minimal repair costs.

The inspection of the results in Tables 2 and 3 reveals that the higher of any of the two costs due to the perfect repair and the minimal repair, the greater, $T^{\star}$, that is, the less frequent the inspection. Moreover higher minor repair costs imply a smaller accumulated number of failures previous to the perfect repair whereas increasing the perfect repair costs causes the opposite effect on $N^{\star}$.

\section{Acknowledgments}

This work has been supported by research Project MTM2007-63683 from the Spanish Ministry of Education.

The authors wish to thank two anonymous referees for his valuable comments.

\section{References}

[1] M. Brown and F. Proschan, "Imperfect repair," Journal of Applied Probability, vol. 20, no. 4, pp. 851-859, 1983.

[2] T. Nakagawa and K. Yasui, "Optimal policies for a system with imperfect maintenance," IEEE Transactions on Reliability, vol. 36, no. 5, pp. 631-633, 1987. 
[3] F. G. Badía and M. D. Berrade, "Minimal repair model for systems under both unrevealed minor failures and unrevealed catastrophic failures," International Mathematical Forum, vol. 2, no. 17-20, pp. 819-834, 2007.

[4] F. G. Badía and M. D. Berrade, "Optimal inspection of a system under unrevealed minor failures and revealed catastrophic failures," in Safety and Reliability for Managing Risk, C. Guedes Soares and E. Zio, Eds., vol. 1, pp. 467-474, Taylor and Francis, London, UK, 2006.

[5] F. G. Badía and M. D. Berrade, "Optimum maintenance of a system under two types of failure," International Journal of Material \& Structural Reliability, vol. 4, no. 1, pp. 27-37, 2006.

[6] R. I. Zequeira and C. Bérenguer, "Optimal scheduling of non-perfect inspections," IMA Journal of Management Mathematics, vol. 17, no. 2, pp. 187-207, 2006.

[7] Y. L. Zhang, R. C. M. Yam, and M. J. Zuo, “A bivariate optimal replacement policy for a multistate repairable system," Reliability Engineering and System Safety, vol. 92, no. 4, pp. 535-542, 2007.

[8] G. J. Wang and Y. L. Zhang, "Optimal periodic preventive repair and replacement policy assuming geometric process repair," IEEE Transactions on Reliability, vol. 55, no. 1, pp. 118-122, 2006.

[9] J. K. Vaurio, "Availability and cost functions for periodically inspected preventively maintained units," Reliability Engineering and System Safety, vol. 63, no. 2, pp. 133-140, 1999.

[10] A. Biswas, J. Sarkar, and S. Sarkar, "Availability of a periodically inspected system, maintained under an imperfect-repair policy," IEEE Transactions on Reliability, vol. 52, no. 3, pp. 311-318, 2003.

[11] S. Ross, Introduction to Probability Models, Academic Press, San Diego, Calif, USA, 8th edition, 2002.

[12] F. G. Badía, M. D. Berrade, and C. A. Campos, "Optimization of inspection intervals based on cost," Journal of Applied Probability, vol. 38, no. 4, pp. 872-881, 2001.

[13] T. Nakagawa, "Periodic and sequential preventive maintenance policies," Journal of Applied Probability, vol. 23, pp. 536-542, 1986. 


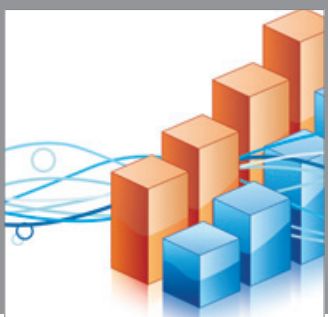

Advances in

Operations Research

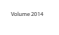

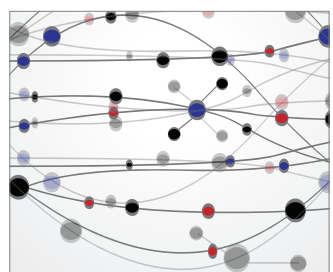

\section{The Scientific} World Journal
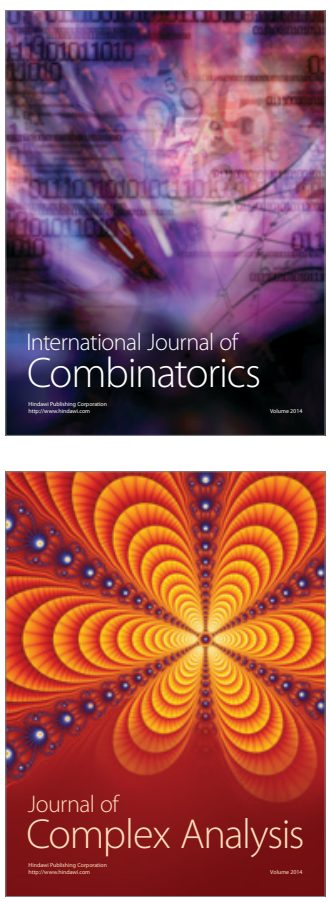

International Journal of

Mathematics and

Mathematical

Sciences
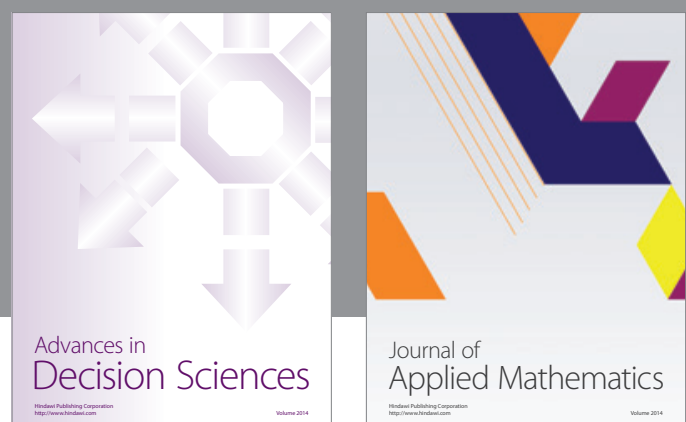

Journal of

Applied Mathematics
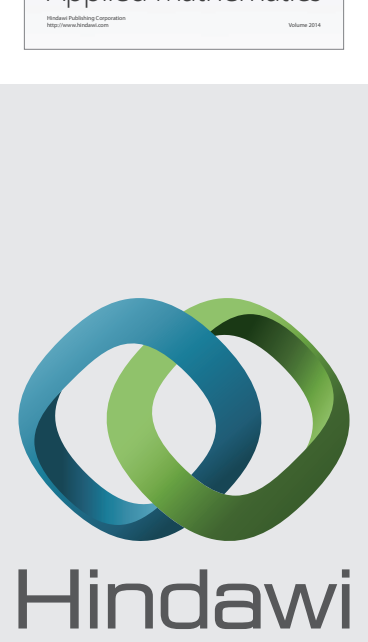

Submit your manuscripts at http://www.hindawi.com
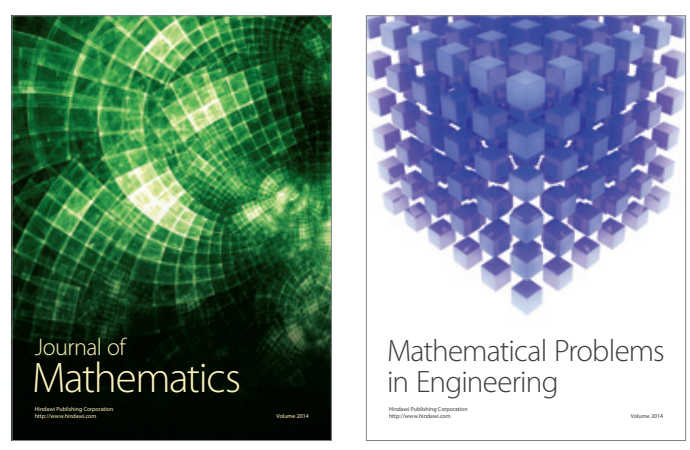

Mathematical Problems in Engineering
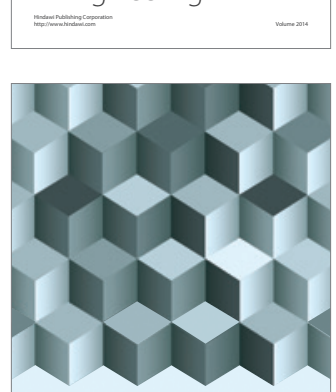

Journal of

Function Spaces
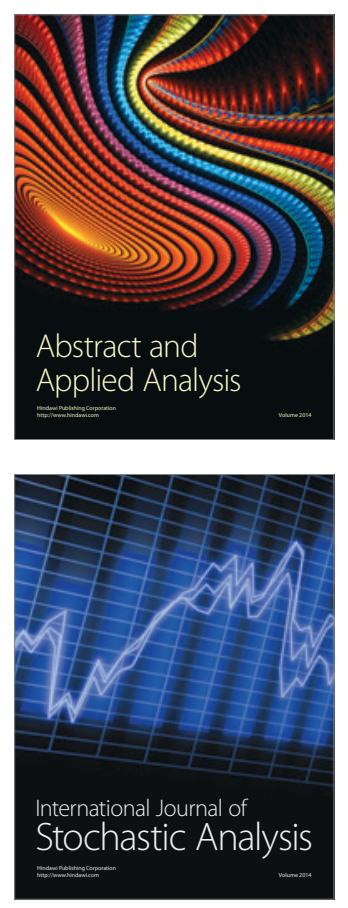

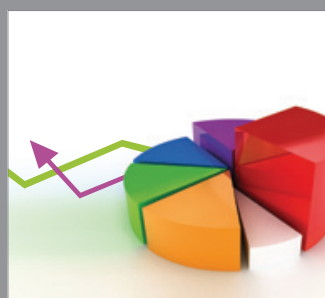

ournal of

Probability and Statistics

Promensencen
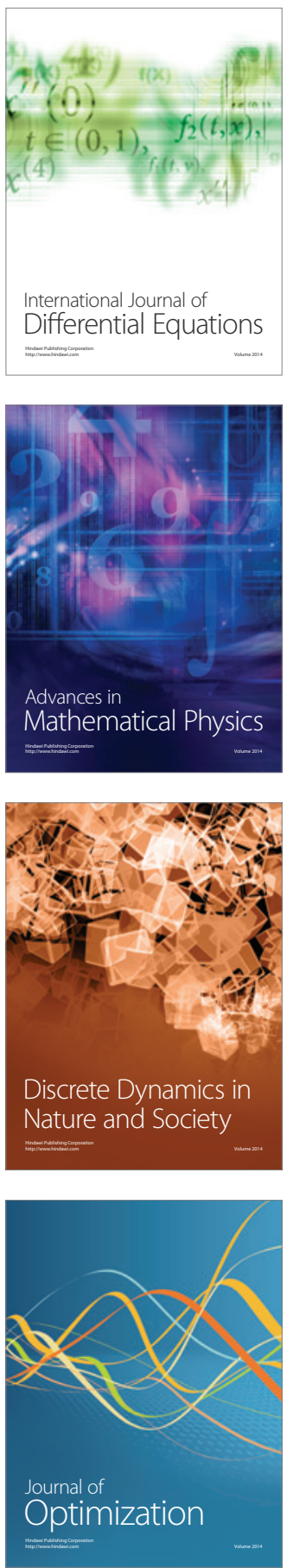